\section{Urologia \\ Internationalis}

\section{Polytimi Leonardou Sofia Gioldasi Paris Pappas}

Department of Radiology, Laikon General Hospital of Athens, Athens, Greece

\title{
Percutaneous Management of Ureteral Stenosis of Transplanted Kidney: Technical and Clinical Aspects
}

Key Words

Kidney $\cdot$ Transplant $\cdot$ Ureteral stenosis $\cdot$ Percutaneous nephrostomy $\cdot$ Endoprothesis

\begin{abstract}
In the field of renal transplantation, advances in the management of graft rejection have led to improved graft and patient survival rates, however other types of complications have now become more apparent, e.g. vascular or urological. The most common urological complications following renal transplantation are ureteral stenosis or obstruction, constituting a significant problem of the renal graft's survival. The most important aspects concerning these complications are early diagnosis and prompt treatment since any delay in their management may lead to renal graft dysfunction or even graft loss. Developments in interventional radiology have provided minimally invasive means to treat urological complications with low complication rates. Herein we review the literature in order to evaluate the efficacy of percutaneous management of ureteral stenosis regarding its safety, immediate and long-term results, complications as well as rate of recurrence.

Copyright $\odot 2011$ S. Karger AG, Basel
\end{abstract}

\section{Introduction}

Urological complications including urinary leaks and ureteric obstruction represent the most important subgroup of nonvascular problems. As recorded in most recent studies, their incidence ranges from 3 to $8 \%[1,2]$. The traditional method of treatment of these complications has been surgical revision, which entails an increased risk of graft loss. With the development of percutaneous methods of treatment, interventional radiology offers a viable alternative to surgery, with lower complication rates.

Ureteric obstructions that are generally classified as early ( $<3$ months) and late ( $>3$ months) depending on when they occur in relation to the time of transplantation, have various causes. Nevertheless, as many studies highlight the significant differences between them, in practice early stenosis tends to be a result of mechanical causes, such as kinks, edema, blood clots or restrictive submucosal tunnel, whereas late stenosis is provoked by generalized or focal fibrosis resulting from ischemia or rejection [3,4]. Consequently, the majority of early obstructions are likely to resolve with percutaneous man-

\section{KARGER}

Fax +41 613061234 E-Mail karger@karger.ch www.karger.com
Paris Pappas, $\mathrm{MD}, \mathrm{PhD}$

Radiology Department

Laikon General Hospital of Athens

17 Ag. Thoma str., GR-11527 Athens (Greece)

Tel. +30 6944606 625, E-Mail pappasparis@ hotmail.com 
agement, while late obstructions are prone to recurrence due to the fibrotic surrounding environment that cannot be eliminated by any procedure. The most common location of obstruction is at the distal ureter and ureterovesical junction, and it seems that ischemia resulting from problems in operative technique during harvesting or from a high dose of immunosuppression is a major etiological factor for these segmental stenoses $[3,5-7]$.

Percutaneous Ureteral Endoprothesis: Technical and Clinical Aspects

Clinical, laboratory and imaging manifestations of obstructive uropathy, commonly used for proper diagnosis, are tenderness or discomfort over the kidney, decrease in urine output, elevated serum urea and creatinine values, hydronephrosis on renal scan, pelvicalyceal dilatation on ultrasound and relatively normal blood flow on color Doppler ultrasound. There should be high suspicion for this complication in order to be differentiated from rejection, since a delay in accurate diagnosis and inappropriate antirejection therapy may result in loss of the transplanted kidney as well as increased patient morbidity and mortality.

Percutaneous management of ureteral stenosis consists of percutaneous nephrostomy, balloon dilatation, insertion of the double-J stent and rarely metallic stent placement. In particular, although there is technical diversification among interventional radiologists according to each one's experience, a commonly adopted procedure includes puncture of a calyx by a 22 -gauge needle under ultrasonographic guidance. When the needle tip is within the collecting system (fig. 1a), urine is aspirated and contrast medium is injected. A short 0.18 -in guidewire is inserted through the Ciba needle (fig. 1b) over which a three-part system sheath is advanced into the renal pelvis. Anatomical information and an assessment of the rate of flow of contrast down the ureter into the bladder are provided from antegrade pyelograms. Afterwards, when there are no signs of hematuria, pain or infection, selective catheterization of the stenotic area follows with the use of angiographic catheters and hydrophilic guidewires. Finally, if it is possible, a double-J ureteral stent is inserted over the guidewire into the bladder for implantation to the stenotic area. In case that the stent cannot pass the stenotic area, dilatation with an angioplasty catheter balloon proceeds before stent placement (fig. 1c). A percutaneous nephrostomy tube is placed at the end (fig. 1d), which usually is removed $48 \mathrm{~h}$ later, as long as the urine has been cleared of any blood and the patency of the stent is confirmed.

Metallic stent placement is an alternative to surgery when double-J endoprostheses does not give a long-lasting result, but it has not yet been generally accepted $[8,9]$ (fig. 2).

\section{Discussion}

After careful and thorough investigation of the literature, we have assumed that the technical success rate of percutaneous management is significantly high (from $58 \%$ up to $95 \%$ ) resulting in amelioration of renal graft function $[7,10,11]$. In a recent and large study conducted by Zavos et al. [1], 96 (6.3\%) patients with urological complications out of 1,525 patients who had undergone renal transplantation were recorded over a 25 -year period. The authors classified urological complications according to the mechanism and the site of urinary tract involvement: graft ureteropelvic obstruction/stenosis was observed in $12.5 \%$ of the cases, ureteral obstruction/stenosis took place in $28.1 \%$, ureterovesical anastomosis obstruction/ stenosis occurred in $30.2 \%$, and $26 \%$ of the patients suffered from obstruction due to urinary leakage or lymphocele, while a small group of patients (3.1\%) had obstructions due to other etiological factors. Surgical intervention was employed in 49 of the 96 urological complications, while the remaining ones were managed either conservatively or by minor invasive procedures. Compensation of renal graft dysfunction was achieved in the $93.8 \%$ of the cases, highlighting the significant role of early diagnosis and prompt treatment of urological complications in the renal graft's survival.

Moreover, in a relatively recent study by Aytekin et al. [12], 19 patients with transplanted kidney suffering from ureteral stenosis were divided into early $(n=9)$ and late $(n=10)$ obstruction groups, and the reported clinical success rate was $100 \%$ for the early and $90 \%$ for the late obstruction group respectively. The majority of the patients were treated percutaneously by means of nephroureterostomy, balloon dilatation and double-J stent insertion, apart from 2 patients with early obstruction who did not experience balloon dilatation and 1 patient with late obstruction and urolithiasis who was treated with nephrostomy and basket extraction without any failure. As the authors notice, there is only a slight difference in longterm outcomes with regard to recurrence rates $(44 \%$ for early and $40 \%$ for late) between the two groups, indicating that the timing of the obstruction does not affect the 

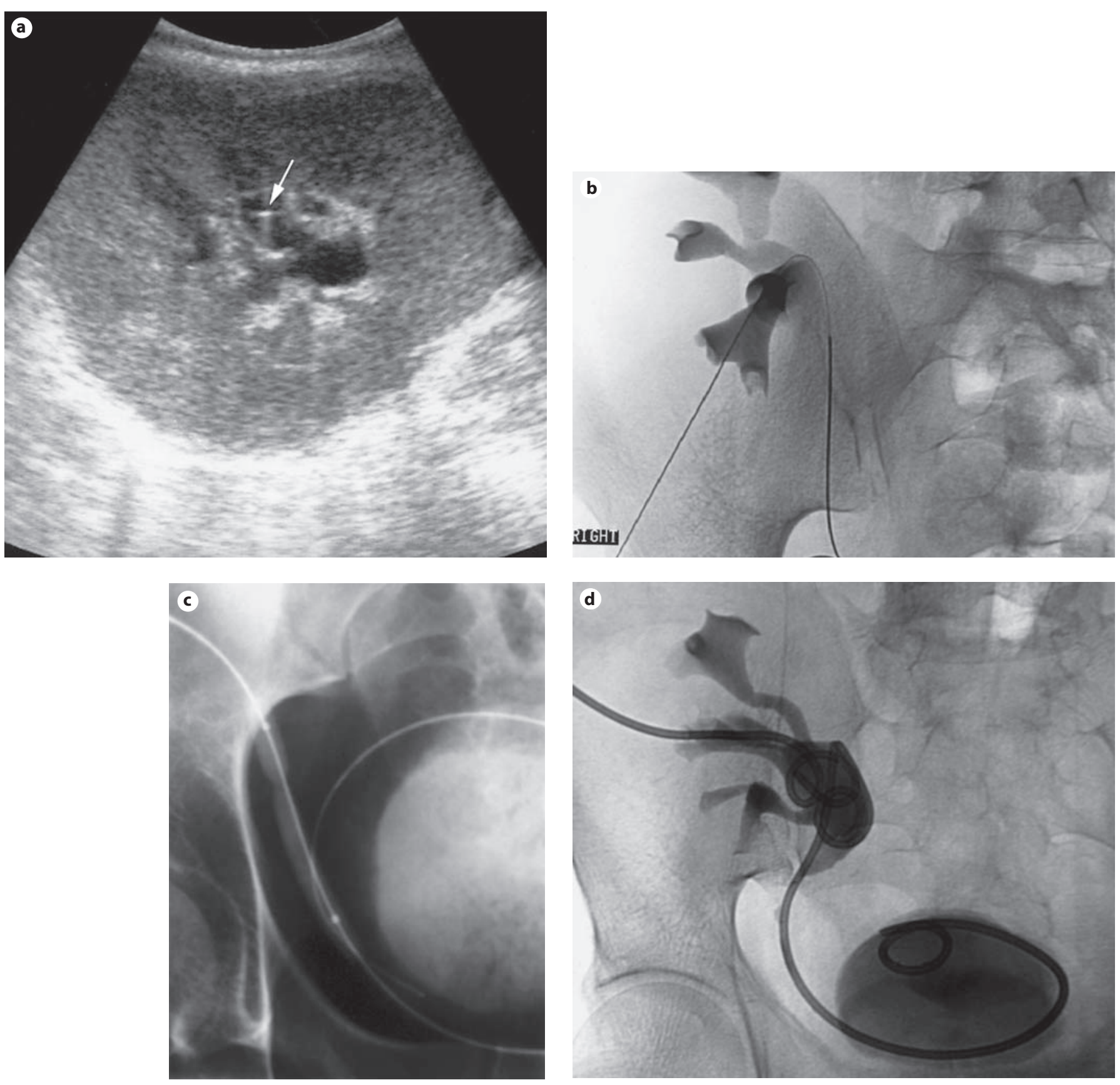

Fig. 1. a Percutaneous calyceal puncture under ultrasonographic guidance (arrow: tip of the needle). b Post contrast injection into the collecting system, through the needle placement of a 0.18 -in guidewire. $c$ Over the wire a balloon dilatation of the ureteral stenosis. d Percutaneous placement of an 8-Fr 24-cm plastic double-J ureteral endoprosthesis and a nephrostomy catheter.

success rate. However, this concept has not been widely verified since the results from many series are controversial. In a less recent retrospective analysis by Bhagat et al. [4], $69 \%$ of the patients who suffered from obstructive uropathy 3 months after transplantation were success- fully treated with the percutaneous procedure, whereas only $33 \%$ of the patients who developed obstruction later had successful treatment revealing the more poor prognosis of late obstruction due to ischemia. 


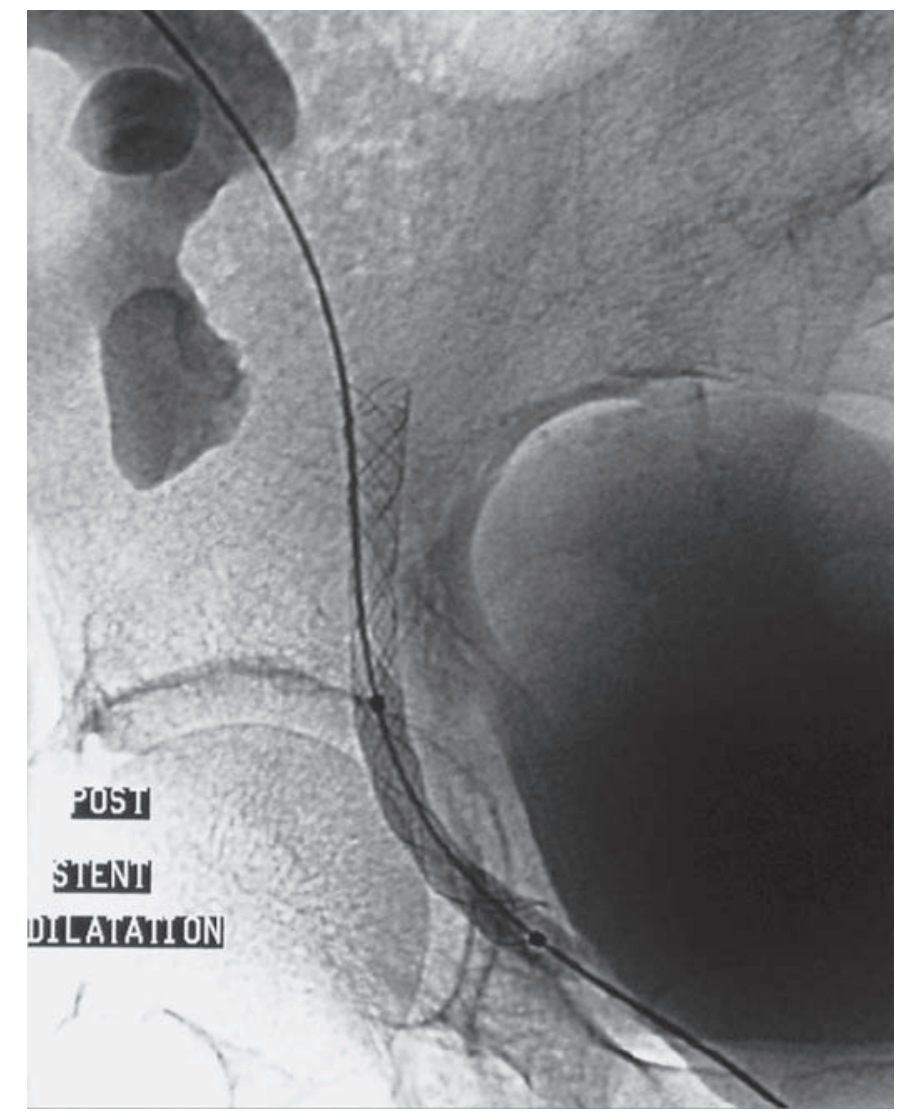

Fig. 2. Post metallic stent balloon dilatation of the ureter in a very tight stenosis. This was the third percutaneous attempt to fix the ureter because of the patient's denial to undergo a surgical operation.

With regard to procedure-related complications, most studies mention minor complications without permanent damage to the transplanted kidney. The overall complication rate reported by Bhagat et al. [4] was $27 \%$ (12 of 45 patients): 7 patients developed infections which resolved with antibiotics, 2 patients experienced urinary leakage at nephrostomy treated adequately by replacing the nephrostomy catheter with another of larger diameter, stent kinking that necessitated antegrade percutaneous replacement occurred in 1 patient and another patient suffered from malposition of the proximal aspect of the ureteral stent during removal of the nephrostomy tube and was treated successfully with antegrade percutaneous replacement of the stent, and finally in 1 patient displacement of the stent into the bladder occurred which was easily removed under cystoscopic guidance. Thus, percutaneous management of ureteral stenosis ameliorates graft function with a low complication rate and low risk of the graft's survival.
Other recent studies highlight the concept of prolonged stenting in the treatment of obstructive uropathy challenging many series that issue stent replacement 3 months after intervention and record success rates from 39 to $70 \%$ [13]. Based on the reasoning that 12 months after stent placement its lumen would have become inactive due to encrustation and in addition the stenotic area would have been wide enough to permit extraluminal flow, Pappas et al. [14] decided to remove the stent from 10 patients at this point (>12 months). After a follow-up period of 51 months, the long-term results were significantly encouraging since 9 out of 10 patients had stable renal function without obstruction (success rate $90 \%$ ), confirming the estimation that prolonged stenting carries neither complications nor patient discomfort.

As evidenced from the review of literature, percutaneous management of ureteral stenosis provides a viable, effective and safe alternative to open surgery, which is associated with higher morbidity and mortality rates [1517]. In one large review by Kashi et al. [16] that compared the efficacy between stent placement and surgery for treatment of obstructive uropathy, the advantage of percutaneous management was indicated since $100 \%$ graft survival was observed in those patients treated with stents whereas $87 \%$ graft survival was noticed in those treated with surgery.

\section{Conclusion}

In conclusion, we suggest that the percutaneous approach is the first-line treatment of choice for ureteral obstruction following kidney transplantation as it shows a low risk for the graft's survival, ameliorates renal function and shows low immediate and long-term complication rates. Contrary to these facts, the recurrence rate after treatment is relatively high but longterm urinary patency is accomplished with further percutaneous methods. Surgery should be reserved only for patients who fail to benefit from interventional treatment for its higher rate of graft dysfunction, loss and recurrence. 


\section{References}

1 Zavos G, Pappas P, Karatzas T, Karidis NP, Bokos J, Stravodimos K, Theodoropoulou E, Boletis J, Kostakis A: Urologic complications: analysis and management of 1,525 consecutive renal transplantations. Transplant Proc 2008;40:1386-1390.

-2 Steeter EH, Little DM, Cranston DW, Morris PJ: The urological complications of renal transplantation: a series of 1,535 patients. BJU Int 2002;90:627-634.

3 Kaskarelis I, Koukoulaki M, Georgantas T, Bairamidis E, Kokkinos C, Ieronymou M, Vougas V, Drakopoulos S: Ureteral complications in renal transplant recipients successfully treated with interventional radiology. Transplant Proc 2008;40:3170-3172.

4 Bhagat VJ, Gordon RL, Osorio RW, LaBerge JM, Kerlan RK, Melzer JS, Bretan P, Wilson MW, Ring EJ: Ureteral obstructions and leaks after transplantation: outcome of percutaneous antegrade ureteral stent placement in 44 patients. Radiology 1998;209: 159-167.

5 Bachar GN, Mor E, Bartal G, Atar E, Goldberg N, Belenky A: Percutaneous balloon dilatation for the treatment of early and late ureteral strictures after renal transplantation: long-term follow-up. Cardiovasc Intervent Radiol 2004;27:335-338.
-6 Garrafiello G, Lagana D, Mangini M, Cuffari S, Cafaro T, Recaldini C, Genovese E, Fugazzola C: The role of interventional radiology in the management of kidney transplant complications. Radiol Med 2005;110:249261.

7 Shivde SR, Date J, Dighe TA, Joshi PM: Unusual causes of obstruction to transplant ureter. Saudi J Kidney Dis Transpl 2010;21: 310-313.

-8 Maan Z, Patel D, Moraitis K, El-Husseiny T, Papatsoris AG, Buchholz NP, Masood J: Comparison of stent-related symptoms between conventional double-J stents and a new-generation thermoexpandable segmental metallic stent: a validated questionnairebased study. J Endourol 2010;24:589-593.

$\checkmark 9$ López-Huertas HL, Polcari AJ, Acosta-Miranda A, Turk TM: Metallic ureteral stents: a cost-effective method of managing benign upper tract obstruction. J Endourol 2010;24 483-485.

10 Burgos FJ, Pascual J, Marcen R, Garcia-Navas R, Garcia IG, Alarcon C, Gomez V, Garcia-Ortells D, Tabernero G, Ortuno J: Selfexpanding metallic ureteral stents for treatment of ureteral stenosis after kidney transplantation. Transplant Proc 2005;37: 3828-3829.
11 Peregrin J, Filipova H, Matl I, Vitko S, Lacha $\mathrm{J}$ : Percutaneous treatment of early and late ureteral stenosis after renal transplantation. Transplant Proc 1997;29:140-141.

12 Aytekin C A, Boyvat F, Harman A, Ozyer U, Colak T, Haberal M: Percutaneous therapy of ureteral obstructions and leak after renal transplantation: long-term results. Cardiovasc Intervent Radiol 2007;30:1178-1184.

-13 Lojanapiwat B, Mital D, Fallon L, Koolpe H, Raja R, Badosa F, Po C, Morris M: Management of ureteral stenosis after renal transplantation. J Am Coll Surg 1994;179:21-24.

14 Pappas P, Stavrodimos G, Adamakis I, Leonardou P, Zavos G, Constantinides C, Kostakis A, Giannopoulos A: Prolonged ureteral stenting in obstruction after renal transplantation: long-term results. Transplant Proc 2004;36:1398-1401.

15 Minnee RC, Bemelman FJ, Laguna Pes PP, ten Berge IJ, Legemate DA, Idu MM: Effectiveness of a 5-day external stenting protocol on urological complications after renal transplantation. World J Surg 2009;33:27222726

16 Kashi SH, Lodge JP, Giles GR, Irving HC: Ureteric complications of renal transplantation. Br J Urol 1992;70:139-143.

$\checkmark 17$ Mongha R, Kumar A: Transplant ureter should be stented routinely. Indian J Urol 2010;26:450-453. 